\title{
Research on Soil Testing and Formula Fertilization System Based on Wireless Sensor Network*
}

\author{
Duan Yunpeng \\ School of information technology, Jilin Agricultural University, Changchun, China \\ duanyunpengjoan@sohu.com
}

Keywords: Soil testing, formula fertilization, wireless sensor network, coverage technology, soil nutrient detection, data fusion technology.

Abstract: The basic problem of agricultural production is to improve the production and quality of agricultural products. Fertilization is the main means to increase the yield of crops. However, at present, in order to improve the yield, the agricultural production in our country has long been in order to improve the output, and the fertilizer is used blindly, especially in the various kinds of chemical fertilizer. The waste of fertilizer also leads to the serious decline of the quality of the agricultural products. In the long run, the mixed fertilizer will pollute the environment more, which makes the nutrient unbalance and no regenerative capacity of the land. It has seriously affected the stability and improvement of agricultural productivity. This study combines soil testing technology and wireless sensor technology to monitor the soil nutrients, moisture, $\mathrm{pH}$ and microbes through wireless sensor, then conduct fusion and analysis on the collected data, and extract effective data. It can provide favorable data basis for soil testing and formulated fertilization technology, so as to improve the current predicament.

\section{Introduction}

The wireless sensor network combines sensing technology, embedded technology, network technology, radio frequency technology, distributed intelligent information processing technology, to realize its miniaturization or miniaturization, integration, multi-function, systematization and networking, especially the design of ultra-low power system which is unique to the sensor network. In the field, the soil information is monitored by wireless sensor network, the soil fertility information is collected, data fusion is realized by the depth learning model of the collected data, and data support is provided for the soil testing formula fertilization technology. In view of the problem of poor field conditions and inconvenient power supply, each node of WSN equipment uses solar power supply and uses CN3722 to design the corresponding charging circuit to realize the selfsupply of the node energy. The system can monitor the atmospheric temperature and humidity, soil temperature and moisture content, wind speed, wind direction and light intensity of seven environmental parameters, and upload the data to remote server to realize the digital and intelligent 
management of farmland. The realization of this system can reduce the cost of agricultural production and increase efficiency, and can be used as a reference for promoting the process of "precision agriculture" in China.

\section{System design}

The wireless sensor network combines sensing technology, embedded technology, network technology, radio frequency technology, distributed intelligent information processing technology, to realize its miniaturization or miniaturization, integration, multi-function, systematization and networking, especially the design of ultra-low power system which is unique to the sensor network[1]. In the field, the soil information is monitored by wireless sensor network, the soil fertility information is collected, data fusion is realized by the depth learning model of the collected data, and data support for soil testing formula fertilization technology is provided.

\subsection{System structure design}

The wireless sensor network (WSN) is based on a large number of integrated sensors deployed in the regional farmland to collaborate in real-time sensing, monitoring the information of soil, weather, crops and so on[3]. The information is processed intelligently through embedded system, and the information is transmitted to the diagnosis decision center through the self-organizing wireless communication network, the remote monitoring and management of soil information can be realized. As shown in Fig. 1.

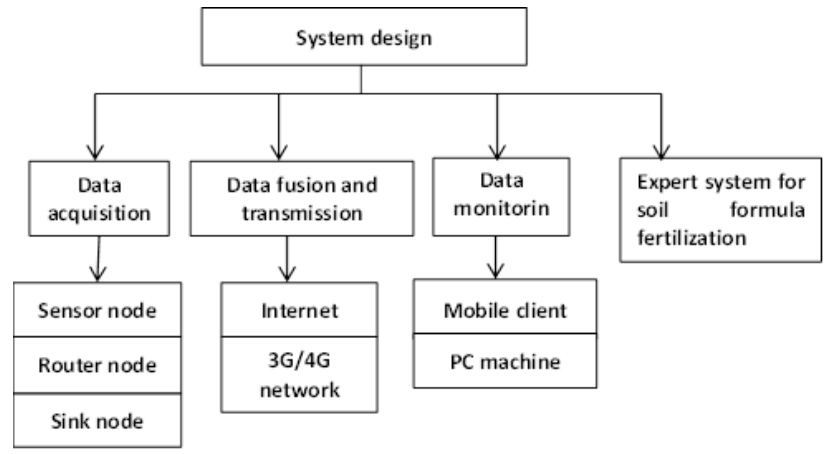

Fig.1. System structure diagram

\section{2 system hardware design}

The template is used to format your paper and style the text. All margins, column widths, line spaces, and text fonts are prescribed; please do not alter them. You may note peculiarities. For example, the head margin in this template measures proportionately more than is customary. This measurement and others are deliberate, using specifications that anticipate your paper as one part of the entire proceedings, and not as an independent document. Please do not revise any of the current designations.

\subsubsection{Soil fertility sensor}

Soil nutrients include N, P, K, Ca, Mg, S and other 13 elements and organic matter content. Soil nutrients mainly come from soil, followed by precipitation, groundwater and so on. The abundance of soil nutrients is directly related to the growth of crops. Appropriate soil nutrient content can increase crop yield and shorten the growth cycle of crops. It is a reference standard for agricultural 
researchers to study agricultural products, and the requirement for different nutrients in different conditions is put forward. A sensor for measuring soil fertility, including a controller, a soil fertility sensor, a mobile communication module, a positioning module, and a soil fertility sensor located at the bottom of the probe, including a sensor shell and a probe shell [4].

\subsubsection{Light temperature sensor}

Solar lighting is the most basic energy source of agricultural growth. Its intensity and time will directly or indirectly affect the growth and development of crops. The illumination on the surface of light is defined as the luminous flux on the unit area[5]. The system uses high sensitivity illuminance detection probe, the signal is stable and the precision is high. It has wide measuring range, good linearity and easy installation.

\subsubsection{Soil moisture sensor}

Soil moisture content (water content) is an important index to reflect soil moisture. It is the basic condition for plant roots to absorb nutrients. Meanwhile, the evaporation of soil moisture also provides support for photosynthesis of plant leaf surface. Soil temperature is the surface temperature of soil, and suitable soil temperature is beneficial to the promotion of root nutrition. Aspiration can enhance the ability of light cooperation. On the contrary, soil temperature is too high or too low, which will inhibit root development and crop growth. The soil temperature is slow in time so refine on words, sensor detection range is wide enough, and the precision is high enough.

The system adopts the soil moisture temperature output sensor, which is a new generation of soil moisture and temperature two in one sensor. It can measure soil moisture and temperature at the same time, and calculate the compensation between parameters to improve the detection precision. The exterior of the sensor is encapsulated with epoxy resin material, and the high quality stainless steel probe is used to measure the permittivity. It is more resistant to acid and alkali corrosion [7]. The steel needle is isolated and never electrolyzed. It can be detected under the ground for a long time.

\subsection{System software design}

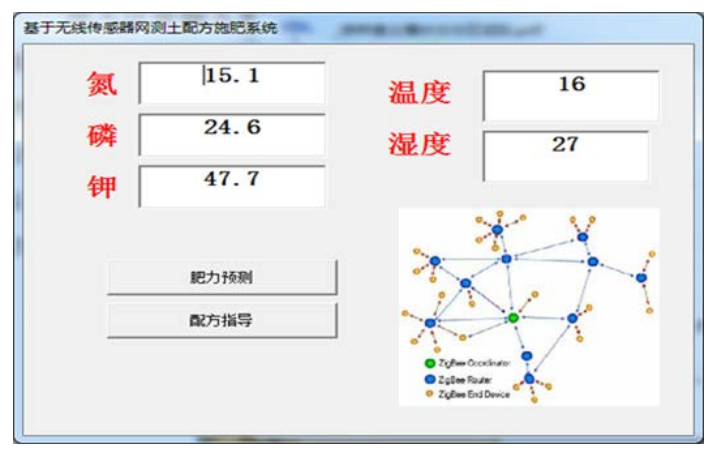

Fig.2. Formula guide interface

The expert system of fertilization is an application of agricultural expert system in agricultural production. A large amount of knowledge of fertilization has been accumulated in the long term production practice, and the application of the expert system of fertilization can also play a greater role in the production practice while storing and protecting this valuable knowledge. The fertilization decision system uses different mathematical models to determine the supply and demand of nutrients in different environments, different crops and different stages of fertility, and is the further development of the direction of fertilization. A large amount of complex information, such as 
regional topography, soil type, soil fertility, meteorology, irrigation, crop species and field test results, can be effectively managed and analyzed, which can be effectively managed and analyzed. The decision of fertilizer recommendation and the formulation of regional fertilizers are the basis for decision making. To a certain extent, fertilization expert system can replace agricultural experts to guide farmers to scientifically and rationally fertilize [5]. Therefore, the establishment of expert system for fertilization is of great significance for guiding agricultural production. As shown in Figure 2.

\section{Key technology of system}

\subsection{Coverage technology for Wireless Sensor Networks}

The propagation of wireless signals in farmland is affected by many complex factors. The variety of crops, height, planting density, plant's bald absorption, and scattering wireless information all cause the attenuation of wireless signals. Even the plants of the same crop are responsible for the propagation characteristics of wireless signals at different stages of their growth. The influence is different. According to the irregularity of the signal propagation path, the irregular perception model is established to control the influence of farmland environment changes on the wireless signal transmission in different generation stages, and the perceptual coverage area is divided and adjusted by the method of irregular sensing coverage increment optimization.

When studying the characteristics of irregular sensing models, considering the characteristics of network coverage and application requirements, the following 2 indexes are considered:

\subsubsection{Effective coverage}

The ratio between the coverage area of the effective area in the network and the total area of the target area is defined as effective coverage. In order to accurately analyze the effective coverage, it is necessary to calculate the coverage area.

\subsubsection{Coverage uniformity}

Coverage uniformity can characterize network balance energy load capacity and reflect network redundancy. The less nodes in the network perceive the overlapping area, the better the coverage uniformity will be. In the wireless sensor network, $\mathrm{N}$ sensor nodes are deployed randomly, the density of nodes is relatively large, and the monitoring area is M. In the early stage of the network operation, all the nodes in the network are working state, while the network maintains better connectivity. The effective coverage of wireless sensor networks is closely related to the range of node perception within the monitoring area. In the process of wireless signal transmission, the signal intensity will decrease with the increase of the signal transmission distance. Therefore, the use of regional coverage techniques, such as Figure 3

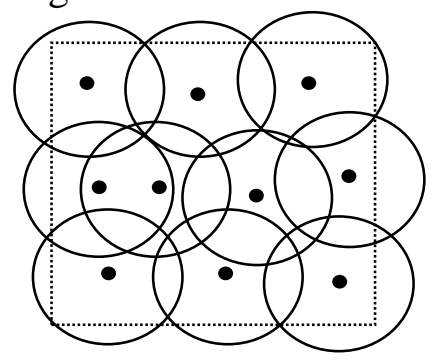

Fig.3.region coverage 


\subsection{Data fusion technology}

The effect of data fusion depends on the classification of feature extraction. Therefore, a WSN feature extraction classification model, SAEM, is introduced in this paper, which is formed by cascading the SAE feature extraction module and the feature classification module containing the classifier. The classifier here can apply the SAEM1 unsupervised classifier and the SAEM2 supervised classifier, in view of the absence of the classifier. Supervised classification algorithm has less $\mathrm{K}$ means parameters, simpler computation and faster convergence, while supervised classifier Softbacks and SAE are trained by layer by layer and overall micro.

The BP algorithm can be used to complete the residual derivation and solve the problem by the gradient descent algorithm, which simplifies the model complexity. Therefore, these two kinds of models are applied to the feature extraction model SAEM. The training of feature extraction classification model is a necessary prerequisite for data classification and fusion of sensor nodes, and the training samples have the classification of unlabeled information. This paper designs a SAEMDA1 and SAEMDA2 unsupervised data fusion algorithm. In order to optimize the comparison, the WSN of the SAEMDA algorithm is the same as that of the same kind of network. Network model:

First, there is only one ID number in the sensor node, which is no longer mobile after random distribution, and the initial energy of nodes is equal and cannot be supplied. Secondly, the sink node is deployed outside the sensing area, with stable location, sufficient energy supply and good storage and computing power. Thirdly, sink nodes can transmit data directly to nodes, while nodes are limited in power, and each node can get their location information

\subsection{Data transmission technology}

\subsubsection{Embedded terminal startup}

The process of terminal starting has automatic boot configuration parameter and self-diagnosis function, completes the inspection and configuration of the sensor, and connects with the network, generally involves a variety of computer networks. The terminal is connected with the terminal access part of the network, and can be divided into finite network, WLAN wireless network, and 4G according to different transmission media, Wireless network and so on.[13]

\subsubsection{Message receiving and transimitting}

After the system starts online, the TCP heartbeat mechanism and the sensor network layer, the transmission network layer are connected, and the packets are sent to the server. Usually set to a interval of 10 seconds, the server sends a heartbeat package to the server, and the server can confirm the terminal online after the server is received; otherwise, the terminal is disconnected and the logical processing is carried out. When the terminal receives the file, it can confirm the connection between the server and the server. After receiving the file of the update command, the terminal should confirm the feasibility of the command and compare it with the logical update command. The identity information identifier is then sent to the server for identification and confirmation.

\subsubsection{Data uploading and downloading}

In the first step, after the server has passed the authentication and logic authentication, the process of data file downloading between the terminal and the server is established. When the terminal sends the download request, the server can feedback the list of data files, which contain the name and 
length of the file. The second step is to download the files one by one according to the file list. The server also records the file transfer process while sending the download command. Using the TCP/IP protocol, the volume and number of files can be downloaded significantly, but some servers usually limit the point to point download thread, and there may be a network anomaly at this time. In this regard, a downloading file size can be set at a certain level, and the data transmission quality can be improved and the transmission time can be shortened by using the function of breakpoint renewal. The third step, after all the data files are downloaded, compare with the file information in the memory, delete the files that are not in the update list, and clean up the redundant information in time.

\subsubsection{Test of legitimacy integrity}

For data transmission, such as interruption, packet loss and so on, after transmission, we should check the legality and integrity of files, avoid falsifying information and tampering with information. In this regard, taking the application of MD5 algorithm as an example, the irreversible check code is used to ensure the security of the file, and the message is sent to the server in time. Confirm that the file information is legal and complete, and then return to the monitoring step of the message, monitor the next update command of the server, and circulate so.

\subsubsection{Exception handling}

In all the steps above, once the network interrupts, downloading failures, monitoring timeouts, error checking, and so on, the terminal will use the exception handling mechanism to correct the error process, return to the message monitor step, and repeat the request.

\section{Conclusion}

In this study, the soil fertility information is collected by sensing nodes such as soil nutrient sensors, soil temperature sensors and soil moisture sensors in wireless sensor networks, and data fusion technology is used to fuse data into effective data, and then the effective data are transmitted to the monitoring terminal and a large number of data are divided. The contents of nitrogen, phosphorus and potassium in soil, as well as the effects of temperature and humidity on soil nutrients, were determined, and the contents of nutrients in soil were determined. The expert system of soil formula fertilization is based on the ratio of these data to the fertilizer program at different periods, and formulating the fertilizer plan and method of fertilization. Through the guidance of the expert system, the soil fertility can be improved in the most suitable period, avoid waste and pollution, and improve the grain yield as well as quality.

\section{Acknowledgment}

This work was financially supported by Jilin Provincial Department of education 13th Five-Year science and technology research project: Research on soil testing and formula fertilization system based on wireless sensor network (JJKH20170295KJ) fund.

\section{References}

[1] Jin Kai Jun, Li Jiang. Data acquisition system based on wireless sensor network. [J]. modern electronic technology, 2017 (40): 72-73.

[2] Zhu Kai, Zhu Huibin, Bai Lizhen, Zhang Jianwei, Xi army, Cao Kegao. ZigBee and C\# based farmland data acquisition system [J]. sensors and Microsystems, 2017 (36): 95-98,

[3] Wang Ren Ran, Pang Junteng, Chen Junjian, Pei Yu, Du Qiliang. Design and implementation of the control system for data acquisition intelligent car based on Arduino control board, [J]. computer technology and automation, 2017 
(36): 66-73.

[4] Zuo Gang, Liu Yanchang, Wang Jianping. Based on Arduino and VI, the design of farmland information wireless collection system [J]. Agricultural Mechanization Research, 2016 (2): 213-217.

[5] Feng Shaozhen. Research and implementation of expert system for soil testing and formulated fertilization [D]. Shandong: Shandong Agricultural University, 2017 (6): 22-31Zhang Zenglin, Yu Xiaoqing. Soil information acquisition system based on wireless sensor network [J]. energy saving irrigation, 2011 (12): 41-43.

[6] Feng Ronghua. Design and implementation of wireless sensor network monitoring system for farmland environment [D]. Fujian: Fujian Agriculture And Forestry University, 51-52.

[7] Sun Yuwen. Research and implementation of farmland environmental monitoring system based on wireless sensor network [D]. Nanjing: Nanjing Agricultural University, 2013,7-9.

[8] Liu Zhaorong. Based on mobile GIS, the high resolution soil fertility information acquisition system in county area [J]. Agricultural Mechanization Research, 2015 (3): 222-225.

[9] Wang Caifen. Construction and application of soil nutrient information system in County orchard based on WEBGIS [D]. Xianyang: Northwest Agriculture and Forestry University, 2016 (5).45-50

[10] Li Guanfeng, Chen Dongmei. Design of Farmland Monitoring System Based on ZigBee technology [J]. Agricultural Mechanization Research, 2013, 35 (11): 107 -10.

[11] Guo Jia, Ma Xinming, Guo Wei, et al. Design of farmland information collection system based on Zig Bee network [J]. Agricultural Mechanization Research, 2013, 35 (11): 65 - 70.

[12] M. Young, The Technical Writer's Handbook. Mill Valley, CA: University Science, 1989.

[13] Chen Wen Yi. Data transfer and input technology of the Internet of things information system [J] electronic technology and software engineering, 2018. (2): 160

[14] Anyuan. Data processing and data transmission technology in wireless sensor networks [D]. Nanjing University of Science and Technology, 2011 (9): 34-45. 